\section{Bioética e atenção básica: \\ um perfil dos problemas éticos vividos por enfermeiros e médicos do Programa Saúde da Família, São Paulo, Brasil}

\author{
Bioethics and primary care: \\ an outline of ethical problems experienced \\ by nurses and physicians in the Family Health \\ Program, São Paulo, Brazil
}

Elma Lourdes Campos Pavone Zoboli 1

Paulo Antonio de Carvalho Fortes 2

\footnotetext{
1 Escola de Enfermagem, Universidade de São Paulo, São Paulo, Brasil.

2 Faculdade de Saúde Pública, Universidade de São Paulo,

São Paulo, Brasil.

Correspondência E. L. C. P. Zoboli Departamento de Enfermagem em Saúde Coletiva, Escola de Enfermagem,

Universidade de São Paulo. Av. Dr. Eneas de Carvalho 419, São Paulo, SP 05403-000, Brasil.

elma@usp.br
}

\begin{abstract}
This is a qualitative empirical study of descriptive ethics conducted with nurses and physicians working in the Family Health Program (FHP) in the city of São Paulo, Brazil. The objective was to identify ethical problems experienced by these professionals, who were asked to record ethical problems they had faced through a short written description of such events. The results revealed ethical problems involving patients and their families, the health team, organization, and the health system. The study addresses ethical issues permeating daily health care practice and requiring immediate solutions, rather than the classical dilemmas usually found in bioethics literature. The peculiarity of ethical problems in primary care may hinder their identification as such and jeopardize the relationships upon which the FHP is built.
\end{abstract}

Bioethics; Primary Health Care; Family Health Program

\section{Introdução}

A implementação do SUS, por representar um processo de mudança na prática da atenção à saúde que exige dos profissionais, gestores e usuários transformações atitudinais e culturais, requer uma reviravolta ética. Assim, para fazer frente ao desafio da sua concretização, faz-se necessário lidar com as questões de ordem ética vivenciadas nos serviços de saúde, especialmente na atenção básica, a qual tem sido preterida pelas reflexões bioéticas.

A atenção básica compreende o conjunto de ações de caráter individual ou coletivo situadas no primeiro nível de atenção dos sistemas de saúde e voltadas para a promoção da saúde, a prevenção de agravos, o tratamento e a reabilitação. O Programa Saúde da Família (PSF) assume um conceito ampliado de atenção básica, avançando na direção de um sistema de saúde integrado que converge para a qualidade de vida das pessoas e de seu meio ambiente. Assim, se a construção do SUS implica uma reviravolta ética, a reorganização da atenção básica pela estratégia do PSF amplia e aprofunda o trajeto desse giro ético, pois sua efetivação não se resume a uma nova configuração da equipe técnico-assistencial, mas a um novo processo de trabalho marcado por uma prática ética, humana e vinculada ao exercício da cidadania.

Com tais questões como pano de fundo e com a finalidade de fazer uma aproximação ini- 
cial às questões éticas que permeiam a atenção básica, foi desenvolvido estudo que teve como objetivos identificar e comparar, a partir de depoimentos de enfermeiros e médicos que atuam no PSF em São Paulo, Brasil, os problemas éticos por eles vivenciados e os fundamentos que utilizam no equacionamento para a tomada de decisão nesses tipos de situação. No presente artigo, relata-se apenas parte dos resultados desse estudo, com o propósito de traçar um panorama dos problemas éticos levantados, comparando-os com estudos estrangeiros similares e com o encontrado na área hospitalar, que tem sido alvo privilegiado da produção em bioética brasileira e internacional.

A sofisticação tecnológica dos hospitais e dos serviços altamente especializados tem sido uma das motivações mais evidentes para o desenvolvimento da bioética, que tem se dedicado mais à reflexão e discussão dos problemas éticos enfrentados pelos profissionais nesse âmbito da assistência, relegando a atenção básica.

Por essa razão, alguns autores 1,2,3,4 têm considerado o atual entendimento da bioética como incompleto, destacando que há fatores indicativos de que os problemas éticos enfrentados na atenção básica podem diferir dos identificados nas demais esferas de atendimento:

(a) os problemas de saúde diferem segundo o nível das ações e dos procedimentos oferecidos; (b) os sujeitos éticos, isto é, os usuários, os familiares e os profissionais de saúde também são diferentes. Pela própria condição da internação, os usuários do hospital têm a capacidade para decisões autônomas comprometida. Os profissionais de saúde, na atenção básica, usualmente visam a objetivos de mais longo prazo a partir da atenção integral e não a apenas sanar um problema pontual;

(c) o cenário em cada tipo de serviço de saúde difere, e isso tem importância na medida em que os problemas éticos emergem do contexto no qual se inserem. Nas unidades básicas de saúde, os encontros com os usuários são mais freqüentes e em situações de menor urgência. A emergência, a imediatidade e a dramaticidade das situações vivenciadas, por exemplo, nos prontos-socorros ou nas unidades de terapia intensiva fazem com que os problemas éticos sejam mais evidentes, tempestuosos e avultados, enquanto, nas unidades básicas de saúde, apresentam-se de maneira mais sutil, passando, muitas vezes, desapercebidos;

(d) as soluções para problemas éticos similares podem diferir, pois, ainda que se observe a mes- ma estrutura de equacionamento ético, os sujeitos e o contexto são distintos, ou seja, os inputs do processo decisório distinguem-se.

\section{Procedimentos metodológicos}

Desenvolveu-se um estudo empírico, qualitativo, de ética descritiva, portanto, de cunho não normativo. Por ética descritiva entende-se a investigação factual da conduta moral por meio de procedimentos e metodologias de natureza científica com vistas a conhecer como as pessoas equacionam e agem, constituindo-se, portanto, tarefa científica e não filosófica. Em bioética, os estudos empíricos, descritivos, têm, entre suas funções, de identificar e caracterizar os problemas éticos que emergem na atenção à saúde 5,6,7,8.

Entre agosto de 2001 e fevereiro de 2002, após a aprovação do projeto pelo Comitê de Ética em Pesquisa da Faculdade de Saúde Pública da Universidade de São Paulo, observando as determinações da Resolução CNS/MS 196/96 (http://conselho.saude.gov.br/docs/Resolucoes/ Reso196de96.doc), foram entrevistados 17 enfermeiros e 16 médicos de unidades básicas de saúde do Município de São Paulo, que contam com PSF implantado. A opção por essas unidades justifica-se pelo fato de o PSF ser a estratégia preconizada pelo Ministério da Saúde para a reorganização da atenção básica no SUS. Como se tratava de pesquisa qualitativa, o critério de representatividade da amostra para o encerramento da coleta de dados foi o da variabilidade, que permitiu abranger a totalidade do problema investigado em suas múltiplas dimensões, ou seja, o da saturação do discurso.

Em entrevistas semi-estruturadas, pediu-se aos profissionais a narração de uma situação na qual considerassem que tinham se defrontado com um problema ético, indicando a solução dada. Em seguida, solicitou-se ao entrevistado que listasse os problemas éticos na situação narrada, segundo sua opinião. Por problema ético compreendem-se, de acordo com Sugarman 9, os aspectos, as questões ou as implicações éticas de ocorrências comuns na prática da atenção à saúde nas unidades básicas, não configurando, necessariamente, um dilema. Após a transcrição dos depoimentos gravados, procedeu-se a análise categorial temática, proposta por Bardin 10. Os discursos foram lidos para a identificação dos problemas apontados, e o conjunto desses formou uma grade 
temática de análise para a leitura transversal de todos os depoimentos, ou seja, cada relato foi relido visando recortá-lo em torno de cada tema-objeto listado.

\section{Resultados e discussão}

Dos 33 depoimentos analisados, vinte (dez em cada grupo) continham relatos de problemas éticos encontrados na tomada de decisão perante os usuários e/ou suas famílias; seis (dois enfermeiros e quatro médicos) listaram problemas éticos em geral do PSF, sem narrar nenhuma experiência específica; dois enfermeiros apontaram situações de discordância e desentendimento entre os integrantes da equipe, sem o envolvimento direto de usuários; três enfermeiros assinalaram rotinas administrativas como potenciais fatores geradores dos problemas; um médico apresentou um caso envolvendo divulgação científica e, por fim, merece destaque o depoimento de um dos médicos que afirmou não ter presenciado, até então, problema ético algum, apenas citou situações de desentendimento entre os profissionais e destes com os usuários sem, entretanto, considerá-las problemas éticos.

Dentre os médicos, registraram-se ainda discursos que apontaram para as dificuldades em definir se as situações que vivenciam representam ou não problemas éticos: "deve ter, mas não tenho visto, todo lugar tem. Mas eu não vi. (....) Em hospital, tá cheio. Quer de hospital?” (Médico 1) “(....) talvez esse dia-a-dia de rotina, pra mim, não seja uma quebra de ética, não seja, é uma coisa normal, que, para mim, é essas encrencas com pacientes (....)" (Médico 2).

Isso denota a sutileza dos problemas éticos vividos na atenção básica. Como pondera Sugarman ${ }^{9}$, os encontros com os usuários nesse nível de assistência caracterizam-se por episódios repetidos e de aparente simplicidade, diferenciando-se das crises bem definidas do hospital que requerem decisões instantâneas, o que pode dificultar o discernimento dos problemas éticos.

Os problemas éticos listados pelos enfermeiros e médicos entrevistados foram agrupados em três categorias, segundo as diferentes dimensões dos relacionamentos no trabalho: problemas éticos nas relações com usuários e família (Tabela 1); problemas éticos nas relações da equipe (Tabela 2); problemas éticos nas relações com a organização e o sistema de saúde (Tabela 3). Representam preocupações do cotidiano da assistência à saúde e não as situações dramáticas próprias do hospital, vindo ao encontro dos achados de estudos realizados em diferentes países, como Estados Unidos 11,12, Israel 13 e Holanda 14.

É óbvio que, com isso, não se quer dizer que os problemas éticos encontrados na atenção básica não ocorram nos hospitais. Por exemplo, o trato desrespeitoso dos usuários, sabidamente, também acontece nos serviços hospitalares, como mostraram estudos sobre as ocorrências registradas por comissões de ética de enfermagem de hospitais das cidades de Ribeirão Preto e São Paulo 15,16. Ao que parece, as situações mais dramáticas e candentes acabam por encobrir as demais, sendo, provavelmente, uma das marcas da diferença entre os dois cenários.

Esses resultados vêm ao encontro de um estudo norte-americano 11 com enfermeiros e médicos de serviços de atenção básica no Kentucky, Estados Unidos, que também registrou, dentre os problemas éticos mais freqüentes, questões como o trato desrespeitoso para com os usuários; a solicitação do usuário por procedimentos desnecessários; a informação inadequada aos usuários; a solicitação de informações por parte da família impondo riscos à preservação da privacidade do usuário e a violação da confidencialidade do usuário.

Também é possível apontar para uma coincidência com alguns dos problemas levantados em estudo com enfermeiros de atenção comunitária de Israel 13, como o conflito entre as necessidades dos usuários e da família; o cuidado a usuários ofensivos; a denúncia de atos incompetentes de médicos ou enfermeiros; o comportamento insultuoso ou rude dos profissionais para com os usuários; a omissão de informação ao usuário por pressões da família; a administração de tratamento errado ou de validade questionável e o constrangimento aos usuários que recusam tratamento.

O PSF caracteriza-se pelo vínculo e responsabilização da equipe para com as famílias de sua área, o que, por certo, traz novas nuanças a um velho problema: o estabelecimento dos limites da relação com o usuário. Alguns entrevistados relataram que chegam a ser "amigos" da família: "você acaba sendo o profissional, o amigo, o enfermeiro da família” (Enfermeiro 11).

Pesquisa realizada por Barros 17 com Equipes de Saúde da Família, no Município de São Paulo, também apontou, como esperado, o acesso às informações que ultrapassam o campo do biológico e do clínico, adentrando aspectos íntimos da dinâmica familiar. Isso, por vezes, causa um certo desconforto ou constrangimento da equipe que não sabe como proceder em tais situações, necessitando ser capacitada para tal.

Estudo com um grupo de enfermeiros que atuava na área de atenção básica em grandes 
Problemas éticos na relação com o usuário e a família.

\begin{tabular}{|c|c|}
\hline Aspectos relativos à (ao) & \\
\hline Relação propriamente dita & $\begin{array}{l}\text { Dificuldade em estabelecer os limites da relação profissional - usuário. } \\
\text { Limites da interferência da equipe no estilo de vida das famílias ou usuários. } \\
\text { Prejulgamento dos usuários dos serviços por parte da equipe. } \\
\text { Desrespeito do profissional para com o usuário. } \\
\text { Atitude do médico frente aos valores religiosos próprios e dos usuários. }\end{array}$ \\
\hline Projeto terapêutico & $\begin{array}{l}\text { Indicações clínicas imprecisas. } \\
\text { Prescrição de medicamentos que o usuário não poderá comprar. } \\
\text { Prescrição de medicamentos mais caros com eficácia igual a dos mais baratos. } \\
\text { Solicitação de procedimentos pelo usuário. } \\
\text { Solicitação de procedimentos por menores de idade sem autorização } \\
\text { ou conhecimento dos pais. }\end{array}$ \\
\hline Informação & $\begin{array}{l}\text { Recusa do usuário às indicações médicas. } \\
\text { Como informar o usuário para conseguir sua adesão ao tratamento. } \\
\text { Omissão de informações ao usuário. } \\
\text { Acesso dos profissionais de saúde a informações relativas à intimidade } \\
\text { da vida familiar e conjugal. }\end{array}$ \\
\hline Privacidade e confidencialidade & $\begin{array}{l}\text { Discussão de detalhes da situação clínica do usuário na sua frente. } \\
\text { Dificuldades para manter a privacidade nos atendimentos domiciliários. } \\
\text { Dificuldades para o agente comunitário de saúde preservar o segredo } \\
\text { profissional. } \\
\text { Compartilhamento das informações sobre um dos membros da família } \\
\text { com os demais. } \\
\text { Não solicitação de consentimento da família para relatar sua história } \\
\text { em publicação científica. }\end{array}$ \\
\hline
\end{tabular}

Tabela 2

Problemas éticos na relação da equipe.

Falta de compromisso dos profissionais que atuam no Programa Saúde da Família.

Falta de companheirismo e colaboração entre as equipes.

Desrespeito entre os integrantes da equipe.

Despreparo dos profissionais para trabalhar no Programa Saúde da Família.

Dificuldades para delimitar as especificidades e responsabilidades de cada profissional.

Questionamento da prescrição médica por parte de funcionário da Unidade de Saúde da Família.

Omissão dos profissionais frente à indicação clínica imprecisa.

Compartilhamento das informações relativas ao usuário e família no âmbito da equipe do Programa Saúde da Família.

Quebra do sigilo médico por outros membros da equipe ao publicarem relatos de casos.

Não solicitação de consentimento da equipe para relatar caso em publicação científica. 
Problemas éticos nas relações com a organização e o sistema de saúde.

\begin{tabular}{|c|c|}
\hline Aspectos relativos à & \\
\hline \multirow[t]{5}{*}{ Unidade de Saúde da Família } & $\begin{array}{l}\text { Dificuldades para preservar privacidade por problemas na estrutura física } \\
\text { e rotinas da Unidade de Saúde da Família. }\end{array}$ \\
\hline & $\begin{array}{l}\text { Falta de estrutura na Unidade de Saúde da Família para a realização } \\
\text { das visitas domiciliárias. }\end{array}$ \\
\hline & $\begin{array}{l}\text { Falta de condições na Unidade de Saúde da Família para atendimentos } \\
\text { de urgência. }\end{array}$ \\
\hline & Falta de apoio estrutural para discutir e resolver os problemas éticos. \\
\hline & $\begin{array}{l}\text { Falta de transparência da direção da Unidade de Saúde da Família } \\
\text { na resolução de problemas com os profissionais. }\end{array}$ \\
\hline \multirow[t]{5}{*}{ Rede de serviços de saúde } & Excesso de famílias adscritas para cada equipe. \\
\hline & Restrição do acesso dos usuários aos serviços. \\
\hline & $\begin{array}{l}\text { Demérito dos encaminhamentos feitos pelos médicos do Programa Saúde } \\
\text { da Família. }\end{array}$ \\
\hline & Dificuldades no acesso a exames complementares. \\
\hline & $\begin{array}{l}\text { Dificuldades quanto ao retorno e confiabilidade dos resultados de exames } \\
\text { laboratoriais. }\end{array}$ \\
\hline
\end{tabular}

cidades do oeste norte-americano indicou que o relacionamento com o usuário tem, para eles, um significado importante, tornando-se mais significativo na medida em que o contato perdura ao longo do tempo ou o usuário apresenta necessidades prementes 12 .

A proximidade na relação levanta questionamentos quanto à interferência da equipe no estilo de vida das famílias ou dos usuários, ou seja, em que medida os profissionais podem ser coercitivos com vistas a conseguir a adesão às terapêuticas propostas e mudanças no estilo de vida: “(...) se a pessoa não enxerga aquilo, apesar de toda a sensibilização, da educação que a gente faz, ela não enxergar aquilo como um fator que vai levar a ter doenças? (....) eu devo invadir essa casa? Nós temos esse direito? É ético esse procedimento?" (Médico 12).

Os usuários devem ter acesso às informações esclarecedoras pertinentes, recebendo orientações ou sendo persuadidos à mudança de estilos de vida não saudáveis. A persuasão é eticamente defensável, porém a coerção não. Da mesma forma, o paternalismo, mesmo requerendo ações de caráter beneficente, é contrário à promoção da autonomia e cidadania das pessoas.

Estudo feito com enfermeiros visitadores na Holanda 18 também identificou essa questão como um problema ético. A exemplo dos profissionais holandeses, os enfermeiros e médicos entrevistados no PSF de São Paulo relataram que, muitas vezes, têm de se adaptar ao modo de vida do usuário para minimizar as conseqüências negativas dos aspectos intrusivos, inevitáveis de seu trabalho. Assim, frente a uma divergência de opiniões com o usuário ou a família, usualmente os profissionais de saúde tentam chegar a um acordo, imediatamente ou no futuro, através da explicação das conseqüências do curso de ação escolhido pelo usuário e das razões pelas quais indicam outra alternativa. Buscam oferecer sugestões e alternativas sem pressionar, tentando ganhar confiança para que os usuários possam manifestar suas objeções, medos ou preocupações e então os ouçam, dêem informação ou os apóiem quando quiserem discutir a questão novamente: “eu sempre explico pro paciente de uma maneira que ele acabe não ficando muito chateado (....) com a equipe (....) porque se ficar chateado (....) vai refletir no nosso trabalho, então a gente tem que tentar sempre com o paciente conversar" (Enfermeiro 14).

Essa tendência de respeitar as escolhas dos usuários e famílias também apareceu em pesquisa com médicos de família dos Estados Unidos, Inglaterra e Canadá, que admitiram discutir o estilo de vida dos usuários e famílias sem interferir na tomada de decisão sobre as mudanças 19.

A dificuldade para manter a privacidade nos atendimentos domiciliários e de definir em que medida as informações privativas dos usuários e famílias devem ser compartilhadas no âmbito da equipe, especialmente em relação ao agen- 
te comunitário de saúde que é um vizinho dessas famílias, são outros problemas decorrentes das peculiaridades que cercam as relações na saúde da família: “(....) às vezes, (....) quando você vai na visita [domiciliária], há outras pessoas” (Enfermeiro 10) “(...) a capacitação dos agentes [comunitários de saúde], um dos temas era ética e sigilo (....) porque eles convivem com os pacientes lá fora (....) estão na casa dos doentes e os doentes estão na rua deles!" (Médico 12).

Alguns estudos realizados com profissionais de saúde e usuários de serviços de saúde na Austrália 4, Estados Unidos 11,12 e Inglaterra 20 também apontaram a manutenção da confidencialidade como um problema ético freqüente na atenção básica. Pesquisa feita na Inglaterra 20 com usuários de consultórios de médicos generalistas e estudo realizado em hospital geral público da região metropolitana de São Paulo 21 encontraram a noção de cuidado vinculada à permissão para acesso às informações de cunho privado, ou seja, os usuários entrevistados, em ambos os estudos, afirmaram que os enfermeiros e médicos que lhes prestavam cuidado deveriam ter acesso ilimitado a seus registros, mas manifestaram restrições para os profissionais de saúde não diretamente envolvidos na sua assistência e para os funcionários dos demais setores dos serviços, como administração, limpeza e segurança.

É necessário ainda considerar, com especial atenção, o lugar da família nessa questão, já que esse aspecto figurou dentre os problemas éticos listados: "você acaba atendendo a família, então, às vezes, você pode entrar nessa armadilha de colocar o caso na família, discutir o caso com a família, e o caso pertence ao paciente" (Enfermeiro 10).

Como mostrou o estudo de Sacardo 21, os usuários entrevistados esperavam que a família partilhasse do segredo de um dos membros em virtude de seu papel "cuidador" que proporciona sentimento de segurança, proteção e diminui a sensação de vulnerabilidade provocada pelo adoecimento. Os familiares foram considerados aliados no processo adoecimento/cura ou hospitalização e, dessa forma, havia pouca expectativa em relação à manutenção absoluta da privacidade das informações no seio da família nuclear. No entanto, os achados da autora também apontaram que, na expectativa dos usuários, a família não deve substituílos na troca de informações com a equipe de saúde, e que a proximidade familiar é algo determinante, pois os usuários estabeleciam um limite entre os familiares mais distantes e os parentes mais próximos. É óbvio que a comparação e o transporte desses resultados para o cenário da saúde da família têm de ser feitos com cautela, pois as informações às quais as equipes têm acesso não dizem respeito exclusivamente a quadros patológicos dos usuários, como pode ser denotado por outros problemas éticos listados pelos entrevistados no presente estudo.

Os problemas éticos na relação com os usuários e família parecem trazer à tona a imprevisibilidade de resultados inerente às relações humanas na saúde, que tende a ser maior na saúde da família pelas peculiaridades que cercam esse relacionamento. De um lado, os usuários e suas famílias buscam a resolução de um problema, de saúde ou não, que consideram importante, trazendo consigo seus segredos, medos, crenças e expectativas. De outro, a equipe, ainda inexperiente para lidar com situações decorrentes da contínua proximidade com os usuários e família, mantém-se presa a procedimentos, normas e rotinas do serviço ou ainda a seu entendimento técnico do que é melhor para os usuários e/ou as famílias.

Os problemas e conflitos na equipe de saúde não são inesperados, especialmente se for considerado que essa, como afirmam Matumoto et al. 22, configura uma rede de relações tecida no cotidiano entre agentes que portam saberes diferenciados e desenvolvem práticas distintas, sendo necessária certa disponibilidade para que reconheçam e respeitem suas diferenças. O peso dos desentendimentos com a equipe para um dos enfermeiros foi tal que chegou a afirmar nunca ter vivido problemas éticos com o usuário, mas somente com os colegas: "então, na verdade, assim, com o paciente, não lembro (....) eu acho mais difícil, às vezes, entre colegas do que entre o paciente" (Enfermeiro 13).

Tal achado guarda consonância com os resultados de estudos estrangeiros e brasileiros realizados tanto em hospitais quanto em serviços de atenção básica, nos quais os profissionais de saúde apontaram seus colegas ou os membros da outra categoria profissional como fontes de problemas éticos, muitas vezes, mais importantes que os usuários e/ou suas famílias 13,14,15,23,24,25.

Vale destacar que somente os enfermeiros relataram situações de conflito e desacordo direto com os médicos. Os últimos, quando fizeram alusão às dificuldades para estabelecer os limites de cada profissional, questionaram o fato do PSF não reconhecer a importância do médico ou a legalidade dos atos feitos pela enfermagem, mas sem trazerem qualquer ocorrência de confronto direto entre enfermeiros e médicos. Isso também foi observado por Udén 
et al. 25 ao pesquisarem enfermeiros e médicos dos departamentos de Medicina Interna e Oncologia do Hospital Universitário de Tromsö, na Noruega. Ao pedirem para que os entrevistados narrassem uma situação de cuidado que fosse eticamente problemática e que tivessem vivido na enfermaria, perceberam que, especialmente nas histórias dos enfermeiros e menos nas dos médicos, havia menções de desacordo entre as duas profissões. Os médicos eram freqüentemente apontados como fontes de conflitos éticos pelos enfermeiros; por outro lado, estes raramente eram mencionados nas narrativas dos primeiros.

Esse estudo não é o único a sugerir essa questão, pesquisas com profissionais de unidades de terapia intensiva nos Estados Unidos 26 e com enfermeiros e médicos de enfermarias de hospitais gerais no Canadá 27 também tiveram achados semelhantes.

No PSF, o desacordo entre os membros da equipe tende a adquirir uma tonalidade nova, pois, como afirmam Silva \& Damaso 28 , a identidade dos sujeitos envolvidos no trabalho fica menos clara. Acrescente-se a isso que a conformação da equipe do PSF, além de incorporar um novo trabalhador, o agente comunitário de saúde, traz também uma mudança na relação numérica dos enfermeiros e médicos. Enquanto, nas unidades de saúde organizadas sob a lógica programática, há usualmente um enfermeiro para, pelo menos, três médicos (um clínico, um pediatra e um ginecologista que atuam, respectivamente, nos programas de saúde do adulto, da criança e da mulher); no PSF, os números se equiparam, com um médico e um enfermeiro em cada equipe. Além disso, no PSF, imputa-se uma carga assistencial importante para o enfermeiro.

Estudo com enfermeiros e médicos da área hospitalar nos Estados Unidos 24 mostrou que, em geral, os enfermeiros assumiam a competência dos médicos, a menos que se provasse o contrário. Em contraste, a preocupação dos médicos pela competência dos enfermeiros era básica para a discussão dos relacionamentos e desacordos, sugerindo que essa não conformava um pressuposto. O que ocorria era justamente o oposto, o conhecimento e o julgamento dos enfermeiros eram assumidos como suspeitos até que se demonstrasse o contrário pela experiência.

Na presente pesquisa, provavelmente pelas peculiaridades do PSF que levam a dificuldades para delimitar as responsabilidades e especificidades de cada profissional, esse questionamento quanto ao preparo e à competência surgiu nos dois sentidos, ou seja, tanto os mé- dicos questionaram a competência dos enfermeiros para o desempenho das tarefas clínicas, como os enfermeiros suspeitaram do preparo dos médicos que, com formação de especialistas, devem fazer o atendimento generalista: “(...) a gente vê (....) muitos médicos que chegam sem capacidade de assumir a população (....) não passa por uma capacitação antes, ele não passa pra ver a saúde da mulher, a saúde da criança, às vezes até um (...) psiquiatra (....)" (Enfermeiro 9) "o sistema que a gente trabalha dá muita liberdade às pessoas que não são médicos (....) você explica, e as pessoas não entendem, porque ela não chega a teu nível intelectual (....)" (Médico 18).

Os problemas éticos nas relações com a equipe trazem à tona as dificuldades crescentes de se delimitar os papéis e funções de cada membro da equipe de saúde em decorrência da incorporação de novos profissionais e das inovações nas propostas assistenciais. Também parece claro que, para fazer frente a isso, os profissionais têm de definir suas atribuições e responsabilidades mutuamente, discutindo as questões de qualificação e competência de maneira conjunta e não cada profissão separadamente, imbuídos de disponibilidade para o diálogo, o respeito às diferenças e sem esquecer que a centralidade da atenção à saúde reside no atendimento das necessidades de saúde do usuário e/ou das famílias, sob risco de comprometê-la se perderem isso de vista.

A conformação do sistema e das organizações de saúde pode se constituir fator gerador de problemas éticos, além de determinar a forma de sua percepção, análise e solução. A estrutura dos serviços, então, parece crucial na geração e no apoio para a resolução dos problemas éticos. Com base em diferentes pesquisas empíricas com enfermeiros e médicos em serviços hospitalares e de atenção básica, Oddi et al. 29 defendem que tais situações representam uma carga bastante pesada para que os profissionais lidem com elas sozinhos.

Na presente pesquisa, um dos problemas éticos levantados foi justamente a falta de apoio estrutural para discutir e resolver questões que suscitam problemas éticos e/ou legais, restando aos entrevistados recorrer aos conselhos dos colegas: “(....) até hoje eu converso com muita gente para ouvir uma opinião, mas ainda eu tô meio dividido, eu não sei se eu fiz certo, se eu fiz errado, se acontecer de novo o que eu vou fazer?" (Médico 10).

Dentre os problemas listados, chama atenção os relativos às dificuldades com os serviços de referência e contra-referência. Sem esquecer as diferenças entre os diversos sistemas de 
saúde, bem como das políticas públicas em cada país, vale citar que as questões de referência e acesso a serviços e procedimentos complementares também apareceram como problemas éticos para os profissionais de saúde em estudos feitos na atenção básica dos Estados Unidos 11,12, Canadá 27 e Holanda 14.

Levantamento acerca dessa temática no sistema de saúde brasileiro, feito por Maeda 30, mostrou a existência de dificuldades estruturais e processuais, dependendo esses mecanismos, muitas vezes, do conhecimento e do relacionamento dos profissionais, sem garantia de acesso dos usuários aos serviços necessários através de uma regulação do próprio sistema com instrumentos formais. Os depoimentos de alguns dos entrevistados expressaram essa realidade: "outra coisa que atrapalha também (....) é a falta de comunicação entre as unidades de saúde e as unidades hospitalares, é uma deficiência de comunicação, mas é uma comunicação burocrática que está deficiente (....) por exemplo: encaminhamentos, via de encaminhamento (....)" (Médico 16).

Segundo Silva \& Damaso 28, o PSF no Município de São Paulo depara-se com insuficiências no funcionamento da retaguarda especializada e nos mecanismos de referência e contra-referência desde a primeira fase de implantação do Projeto Qualis. Para as autoras, parece que essa dificuldade persiste, pois, em estudo recente com médicos, enfermeiros, auxiliares de enfermagem e gerentes locais, identificaram problemas com o fornecimento de medicação e acesso a serviços de referência e contra-referência, tanto ambulatoriais como leitos para internação.

Os problemas éticos na categoria "relações com a organização e o sistema de saúde" guardam uma relação direta com a ética na gestão dos serviços de saúde, deixando patente a dificuldade de, pela própria finalidade das instituições sanitárias, separar a ética dos cuidados de saúde da ética na administração em saúde. Parece claro também que, se os problemas éticos são modulados em sua geração e solução pelas condições de trabalho, seria recomendável a implementação de estratégias para apoiar a condução de tais situações, além da criação de oportunidades para os profissionais de saúde se engajarem em discussões de temas éticos, com vistas a fomentar um ambiente de trabalho sadio, colaborativo e que propicie a excelência técnica e moral da assistência prestada.

\section{Considerações finais}

Os problemas éticos apontados parecem confirmar que, na atenção básica, esses são constituídos, de maneira geral, por preocupações do cotidiano, aspectos éticos que permeiam circunstâncias comuns da prática diária da atenção à saúde e não por situações dilemáticas, merecedoras do destaque midiático, que requerem soluções imediatas, usualmente mais exploradas na literatura bioética. Isso não significa que sejam de menor monta ou importância, e sim que a atenção básica, em comparação à hospitalar, lida com problemas éticos distintos, que, à sua maneira, também são amplos e complexos, ainda que de menor dramaticidade. Essa peculiaridade dos problemas éticos vividos na atenção básica pode levar à dificuldade em identificá-los como tal, pondo em risco a relação vincular que está no cerne do PSF.

Atuar na atenção básica reorganizada pela estratégia PSF requer redirecionamento não só da prática clínica, mas também do equacionamento ético, desfocando-os do hospitalocentrismo e da alta especialização que marcam a conformação do sistema de saúde e a formação dos profissionais e que têm levado a bioética, nas últimas três décadas, a centrar-se nas situações limite, em detrimento das situações do cotidiano.

Isso parece reforçar a necessidade de uma aguçada sensibilidade e compromisso éticos por parte dos profissionais na atenção básica, pois a efetivação do PSF não se resume a uma nova conformação da equipe ou da unidade básica de saúde. Os profissionais que atuam no PSF têm de exercer uma nova prática marcada pela humanização, pelo cuidado, pelo exercício da cidadania e alicerçada na compreensão de que as condições de vida definem o processo saúde-doença das famílias. 


\section{Resumo}

Estudo empírico, qualitativo, de ética descritiva, com enfermeiros e médicos do Programa Saúde da Família (PSF), no Município de São Paulo, Brasil, com o objetivo de identificar os problemas éticos vivenciados por esses profissionais. Tendo sido solicitado aos profissionais que listassem problemas éticos a partir da narrativa de um caso vivido, os resultados apontam para problemas éticos na relação com o usuário e a família, na relação da equipe de saúde e nas relações com a organização e o sistema de saúde. Isto é, aspectos éticos que permeiam circunstâncias comuns da prática diária da atenção à saúde e não situações dilemáticas, que requerem soluções imediatas, usualmente mais exploradas na literatura bioética. Essa peculiaridade dos problemas éticos vividos na atenção básica pode levar à dificuldade em identificá-los como tal, pondo em risco a relação vincular que está no cerne do PSF.

Bioética; Cuidados Primários de Saúde; Programa Saúde da Família

\section{Colaboradores}

E. L. C. P. Zoboli elaborou os originais do artigo, e P. A. C. Fortes os revisou.

\section{Referências}

1. Brody H. Transparency: informed consent in primary care. Hasting Cent Rep 1989; 19:5-9.

2. Gracia D. Bioética clínica. Santa Fé de Bogotá: Editorial El Búho; 1998.

3. Fetters MD, Brody H. The epidemiology of bioethics. J Clin Ethics 1999; 10:107-15.

4. Braunack-Mayer AJ. What makes a problem an ethical problem? An empirical perspective on the nature of ethical problems in general practice. $J$ Med Ethics 2001; 27:98-103.

5. Maliandi R. Ética: conceptos y problemas. Buenos Aires: Editorial Biblos; 1991.

6. Sánchez-González M. La investigación bioética: tipos, funciones y relaciones mutuas. Cuadernos del Programa Regional de Bioética 1998; 6:57-76.

7. Beauchamp TL, Childress JF. Principles of biomedical ethics. 5th Ed. New York: Oxford University Press; 2001.

8. Sulmasy DP, Sugarman J. The many methods of medical ethics. In: Sugarman J, Sulmasy DP, editors. Methods in medical ethics. Washington DC: Georgetown University Press; 2001. p. 3-18.

9. Sugarman J, editor. Ethics in primary care. New York: McGraaw-Hill; 2000. p. xiii-xvi.

10. Bardin L. Análise de conteúdo. Lisboa: Edições 70; 1977.

11. Robillard HM, High DM, Sebastian JG, Pisaneschi JI, Perritt LJ, Mahler DM. Ethical issues in primary health care: a survey of practioners' perceptions. J Community Health 1989; 14:9-17.

12. Viens DC. Moral dilemmas experienced by nurse practitioners. Nurse Pract Forum 1994; 5:209-14.

13. Wagner N, Ronen I. Ethical dilemmas experienced by hospital and community nurses: an Israeli survey. Nurs Ethics 1996; 3:294-304.

14. Van Der Arend AJG, Remmers-Van Der Hurk HM. Moral problems among dutch nurses: a survey. Nurs Ethics 1999; 6: 468-82.

15. Ducati C, Boemer MR. Comissões de ética de enfermagem em instituições de saúde de Ribeirão Preto. Rev Lat Am Enfermagem 2001; 9: 27-32.

16. Freitas GF. Ocorrências éticas com pessoal de enfermagem de um hospital do Município de São Paulo [Dissertação de Mestrado]. São Paulo: Escola de Enfermagem, Universidade de São Paulo; 2002.

17. Barros VL. O informar e o consentir enquanto atributos fundamentais para a consolidação dos direitos do paciente [Dissertação de Mestrado]. São Paulo: Faculdade de Saúde Pública, Universidade de São Paulo; 2000.

18. Gremmen I. Visiting nurses' situated ethics: beyond 'care versus justice'. Nurs Ethics 1999; 6:51527.

19. Hoffmaster CB, Stewart MA, Christie RJ. Ethical decision making by family doctors in Canada, Britain and the United States. Soc Sci Med 1991; 33:647-53.

20. Carman D, Britten N. Confidentiality of medical records: the patient's perspective. Br J Gen Pract 1995; 45:485-8.

21. Sacardo DP. Expectativa de privacidade segundo pessoas hospitalizadas e não hospitalizadas: um estudo bioético [Dissertação de Mestrado]. São 
Paulo: Faculdade de Saúde Pública, Universidade de São Paulo; 2001.

22. Matumoto S, Mishima SM, Pinto IC. Saúde Coletiva: um desafio para a enfermagem. Cad Saúde Pública 2001; 17: 233-41.

23. Pellegrino ED, Hart RJ, Henderson SR, Loeb SE, Edwards G. Relevance an utility of courses in medical ethics: a survey of physicians' perceptions. JAMA 1985; 253:49-53.

24. Prescott PA, Hart RJ, Bowen AS. Physician-nurse relationships. Ann Intern Med 1985; 103:127-33.

25. Udén G, Norberg A, Lindseth A, Marhaug V. Ethical reasoning in nurses' and physicians' stories about care episodes. J Adv Nurs 1992; 17:1028-34.

26. Gramelspacher GP, Howell JD, Young MJ. Perceptions of ethical problems by nurses and doctors. Arch Intern Med 1986; 146:577-8.
27. Oberle K, Hughes D. Doctors' and nurses' perceptions of ethical problems in end-of-life decisions. J Adv Nurs 2001; 33:707-15.

28. Silva JA, Dalmaso ASW. Agente Comunitário de Saúde: o ser, o saber, o fazer. Rio de Janeiro: Editora Fiocruz; 2002.

29. Oddi LF, Cassidy VR, Fisher C. Nurses' sensitive to the ethical aspects of clinical practice. Nurs Ethics 1995; 2:197-209.

30. Maeda ST. Gestão da referência e contra-referência na atenção ao ciclo gravídico-puerperal: a realidade do Distrito do Butantã [Tese de Doutorado]. São Paulo: Escola de Enfermagem, Universidade de São Paulo; 2002.

Recebido em 11/Mar/2004

Versão final reapresentada em 30/Jun/2004

Aprovado em 19/Jul/2004 